\title{
REPERCUSSÕES DA CIRURGIA BARIÁTRICA NOS SINTOMAS DE DEPRESSÃO, ANSIEDADE E QUALIDADE DE VIDA
}

Marion Ribeiro de Medeiros ${ }^{1}$

Resumo: A obesidade é uma doença de origem multifatorial, ocasiona prejuízos fisiológicos, psíquicos e sociais. A cirurgia bariátrica surge como uma solução eficaz, para a perda de peso e controle das doenças comórbidas. O objetivo desse estudo foi avaliar as repercussões da cirurgia bariátrica nos sintomas de depressão, ansiedade e na qualidade de vida no indivíduo obeso, por meio de uma revisão de literatura nas principais bases de dados. Os resultados mostraram que somente a cirurgia bariátrica não soluciona de maneira definitiva a obesidade e suas ressonâncias. Havendo a necessidade de ações interdisciplinares à longo prazo e um olhar biopsicossocial sob o indivíduo após o procedimento.

Palavras-chaves: Cirurgia bariátrica. Depressão. Ansiedade. Qualidade de vida.

\section{REPERCUSSIONS OF BARIATRIC SURGERY ON SYMPTOMS OF DEPRESSION, ANXIETY AND QUALITY OF LIFE}

\begin{abstract}
Obesity is a disease of multifactorial origin, causing physiological, psychic and social damages. Bariatric surgery is an effective solution for weight loss and control of comorbid diseases. The aim of this study was to evaluate the repercussions of bariatric surgery on the symptoms of depression, anxiety and quality of life in obese individuals, through a literature review in the main databases. The results showed that only bariatric surgery does not definitively solve obesity and its resonances. There is a need for long-term interdisciplinary actions and a biopsychosocial look under the individual after the procedure.
\end{abstract}

Keywords: Bariatric surgery. Depression. Anxiety. Life quality.

\footnotetext{
${ }^{1}$ Acadêmica do Curso de Bacharelado em Psicologia da UNICNEC. 


\section{INTRODUÇÃO}

O aumento constante da população obesa em todo o mundo é um desafio à saúde pública (Coutinho, 1999). De origem multifatorial, a obesidade afeta milhões de pessoas, o relatório desenvolvido em 2008 pela Organização Mundial da Saúde (OMS) afirmou que a obesidade alcançou proporções epidêmicas, estimando que 500 milhões de pessoas estariam clinicamente obesas em todo o mundo (Flores,2014), projetando que até 2015 chegaria a 700 milhões (Melo, 2009). No Brasil há um predomínio de 8,9\% em homens e 13,1\% em mulheres, levando ao desenvolvimento de diretrizes para a atenção à saúde, tendo o foco na prevenção e assistência ao portador de obesidade (MINISTÉRIO DA SAÚDE, 2007).

Para Ades e Kerbauy (2002), a origem da obesidade pode ser por uma condição fisiológica, psicológica, social ou situacional. Caracteriza-se por um estado inflamatório crônico que compromete o funcionamento imunológico e metabólico (Leite, Rocha e Neto, 2009). O aumento da massa de gordura corporal é desencadeado por influências genéticas, fatores ambientais, alimentação rica em calorias e sedentarismo (Gayoso et al, 1999), fatores que aumentaram com as mudanças dos hábitos alimentares adotados com a urbanização e a globalização. Flandrin e Montanari (1998) relacionam os hábitos alimentares e a gordura ao contexto social e a cultura, em uma retrospectiva afirmam que a quantidade de comida que se ingeria e o peso que se adquiria definiam o status social de um indivíduo. Contudo, além dos fatores biológicos ou hereditários, atualmente a diversidade de alimentos oferecidos, seja em qualidade ou quantidade, associados ao ato de comer por prazer, possibilita o desenvolvimento de transtornos alimentares.

A obesidade é avaliada pelo cálculo do Índice de Massa Corporal (IMC), sendo divido o peso do indivíduo pelo quadrado de sua altura, que ao apresentar o resultado de 30 a 34,9 $\mathrm{Kg} / \mathrm{m}^{2}$ é considerado Obesidade Grau I, a Obesidade Grau II o resultado é 35 a 39,9 $\mathrm{Kg} / \mathrm{m}^{2}$ e a Obesidade Grau III, ou seja mórbida, o resultado é a partir de $40 \mathrm{Kg} / \mathrm{m}^{2}$ (WHO, 2006). No Brasil cerca de $40 \%$ da população está acima do peso, sendo $28,5 \%$ com sobrepeso. A taxa de mortalidade é 12 vezes maior em indivíduos mórbidos, isto é, que apresentam IMC acima de $40 \mathrm{Kg} / \mathrm{m}^{2}$ (Garrido, 2003). As estratégias adotadas pelos programas de atenção à saúde, que incluem dietas, psicoterapias, atividades físicas e medicamentos específicos muitas vezes não são eficazes para os indivíduos com obesidade mórbida, que no insucesso acumulam frustrações e ansiedades. A cirurgia bariátrica (CB) é utilizada para redução de peso, 
considerada um procedimento radical (Arasaki, 2005), a perda de peso pode atingir até 50\% do inicial em pouco tempo (Pereira, 2003) possibilitando sua manutenção através da adoção de hábitos alimentares controlados por nutricionista, atividade física e apoio psicológico.

Prejuízos físicos, orgânicos e psicológicos, estão presentes na vida do indivíduo obeso. O desenvolvimento de doenças crônicas como diabetes, hipertensão arterial, doenças osteoarticulares, apneia do sono, somados a sintomas de ansiedade e depressão acarretam alterações comportamentais. Juntamente a vivências sociais de exclusão como bullying e preconceitos, que tendem a desenvolver transtornos alimentares e de humor (Almeida, Zanatta e Rezende, 2012; Gayoso et al, 1999). A saúde debilitada e a insatisfação com o próprio corpo são fatores que impulsionam a busca pela cirurgia bariátrica, sendo importante salientar que a percepção que o indivíduo tem de seu próprio corpo influencia em seus pensamentos, sentimentos e na percepção do mundo a sua volta (CASH; PRUZINSKY, 2002).

A cirurgia bariátrica proporciona a perda de peso, redução das doenças que o excesso de peso implica e uma melhora da qualidade de vida (Segal e Fadiño,2002). É um procedimento eficaz, em que o paciente perde peso rapidamente e continua a perder até 24 meses após a cirurgia (Sabiston,1999). Há uma alteração rápida e contínua da imagem corporal exigindo uma reestruturação perceptiva. Cabe ao indivíduo, com apoio multiprofissional readaptar-se a uma nova vida, sendo um processo complexo que envolve aspectos físicos, psíquicos e sociais com resultados totalmente subjetivos. Contudo a eficácia comprovada da cirurgia não garante que futuramente este indivíduo não venha a recuperar seu peso (SILVA, 2011).

\section{FUNDAMENTAÇÃO TEÓRICA}

\subsection{A epidemiologia}

Considerada uma doença crônica, a obesidade é caracterizada pelo excessivo acúmulo de gordura definida por multifatores (WHO, 2000; Diniz e Maciante, 2012), considerados de forma isolada ou combinados, podem ser de origem genética, endócrino, social, econômico, 
psicológico ou ambiental (Rosembaum, 2012). A obesidade é um fator de risco por provocar o aparecimento de inúmeras doenças e comprometer a qualidade e a expectativa de vida (Berti e Caravatto, 2012). Esta condição de risco implica no surgimento de doenças crônicas e de sofrimentos psicossociais (MARCELINO; PATRICIO, 2011).

Mattos (2007) afirma que a obesidade não é uma patologia psiquiátrica, por estar vinculada a causas orgânicas, porém intensamente entrelaçada a fatores emocionais, de ordem psicossomática. Está associada a transtornos psiquiátricos, como depressão e ansiedade em $60 \%$ dos casos e relacionada com transtornos de alimentação em 30\% dos casos (Mello Filho, 2000). Segundo o DSM-V (2014), a obesidade se caracteriza pelo excesso de gordura corporal gerado pelo grande consumo de calorias, durante um longo período, em comparação ao seu gasto. Considerando a constituição singular de cada indivíduo conjugada aos fatores genéticos, fisiológicos, comportamentais e ambientais que contribuem para o seu desenvolvimento, assim a obesidade não é considerada uma doença mental.

No Brasil, estima-se que haja 65 milhões de pessoas com sobrepeso, 14 milhões com obesidade e 4 milhões com obesidade mórbida (Berti e Caravatto, 2012). A obesidade , segundo a OMS, é classificada pelo cálculo do Índice de Massa Corporal (IMC), que se dá pelo peso em quilos $(\mathrm{Kg})$ do indivíduo dividido pelo quadrado de sua altura em metro $(\mathrm{m})$, sendo considerada obesidade grau I, o IMC entre 30 e $34,9 \mathrm{Kg} / \mathrm{m}^{2}$; obesidade grau II, IMC entre 35 e $39,9 \mathrm{Kg} / \mathrm{m}^{2}$ e obesidade grau III, IMC a partir de $40 \mathrm{Kg} / \mathrm{m}^{2}$ (Garrow e Webster, 1985; Anjos,1992). Entendendo como grau I obesidade leve ou sobrepeso, grau II obesidade moderada e grau III obesidade severa ou mórbida

Atualmente o grande crescimento do número de pessoas obesas passou a ser considerado um problema de saúde pública, por ocupar o sistema público de saúde no tratamento das doenças comórbidas e no tratamento da própria redução de peso. A obesidade severa ou mórbida gera fatores de risco como danos cardiovasculares, ortopédicos, endócrinos, dermatológicos e respiratórios (Marcelino e Patrício, 2011). Cabe lembrar que o excesso de peso pode desenvolver alterações psiquiátricas como ansiedade e depressão, que geram prejuízos nas interrelações e laborais (Leal e Baldin, 2007). Em 2011, juntamente com ministros da saúde dos países americanos a OMS redigiu uma declaração sugerindo a luta contra a obesidade através de políticas públicas (CASTRO,2011).

Garrido (2003) alerta que no Brasil, aproximadamente $40 \%$ da população está acima do peso, sendo $10,1 \%$ obesos e $28,5 \%$ sobrepeso. Com relação a taxa de mortalidade, afirma, 
que é 12 vezes maior em obesos mórbidas em relação a pessoas com peso normal, com idade entre 25 e 40 anos. Diante desta realidade, os programas de saúde desenvolvem estratégias para estimular práticas saudáveis, contudo ineficazes para o obeso mórbido, pois exige mudanças alimentares, prática de exercícios físicos e atenção à saúde mental a longo prazo, o que demanda uma motivação constante diante da gravidade da obesidade (SUPLICY, 2005).

\subsection{Implicações biopsicossociais}

A busca pelo peso e o nível ideal de saúde exige uma atenção a nutrição. No Brasil, o aumento da população obesa refletiu no aumento das doenças comórbidas, motivando ações que orientem a redução das deficiências nutricionais da alimentação adotada e visando a prevenção das doenças crônicas. Contudo o foco da prevenção no serviço público de saúde ainda é voltado a doenças infecciosas e carenciais. As doenças crônicas como infarto do miocárdio, diabetes, cânceres, hipertensão estão muitas vezes interligadas e associadas a fatores de risco, por exemplo o diabetes tipo II pode estar associado ao aumento de triglicerídeos. Assim os programas de promoção da saúde visam a adesão às dietas saudáveis e a atividade física com intuito de reduzir os riscos que a obesidade implica (SICHIERI et al, 2000).

São inúmeros os tratamentos para perda de peso, porém o indivíduo com obesidade mórbida dificilmente alcançará o peso idealizado pelos meios tradicionais, gerando frustrações, ansiedade, estresse e até mesmo depressão. Ao avaliar este cenário a cirurgia bariátrica entra como uma solução, a partir dos anos setenta (Marcelino e Patrício, 2011), possibilitando uma redução de peso de $40 \%$ em um ano após o procedimento (SEGAL; FANDIÑO, 2002).

O paciente com obesidade mórbida deve ser considerado como um indivíduo doente, que tem alto risco de morte, que potencialmente terá sua qualidade de vida reduzida e que exigirá um trabalho motivacional antes e após a cirurgia (Björntorp, 2003). Além do critério do IMC ser a partir de $40 \mathrm{Kg} / \mathrm{m}^{2}$ ou acima de $35 \mathrm{Kg} / \mathrm{m}^{2}$ quando associado a doenças com mais de cinco anos de evolução, como diabetes mellitus, o candidato deve apresentar um histórico de falhas diante de tratamentos convencionais para obesidade (Pories, 2003). Segal e Fandiño (2002) afirmam que a presença de transtornos depressivo, bipolar ou psicótico são 
fatores contraindicados, influenciando no bom prognóstico. O mesmo pode ser dito aos pacientes com dependência de etílicos (HSU,1998).

Antes de realizar este procedimento, muitos exames abrangendo múltiplos aspectos são analisados. Uma equipe multidisciplinar formada por enfermeiro, nutricionista, psicólogo, fisioterapeuta, médico cirurgião e outros avaliam as particularidades que implicam na causa da obesidade para traçar um prognóstico (Costa et al, 2009). Segundo o Conselho Federal de Psicologia (2013) o psicólogo ou psiquiatra responsável pela avaliação pré-operatória deve verificar a relação do paciente com substâncias, a presença de quadros psicóticos ou demenciais. Inclusive garantir que o candidato à cirurgia bariátrica, compreenda os riscos e cuidados pós-operatório.

Prejuízos físicos e comportamentais são comuns em indivíduos obesos, normalmente vítimas de discriminações e preconceitos acabam desenvolvendo distúrbios psicossociais (Teichmann et al, 2006). Havendo uma prevalência no transtorno de compulsão alimentar periódica, que se caracteriza pela ingestão de grandes quantidades de comida em um curto espaço de tempo, seguido de sentimento de culpa e falta de autocontrole. Existe uma dificuldade em diagnosticar tal transtorno, contudo a obesidade e a depressão são mais frequentes em quem o tem, exigindo maiores cuidados no pós-operatório (Azevedo, Santos e Fonseca, 2004). A compulsão pode estar vinculada ao transtorno de ansiedade, o ato de comer é usado para amenizar os sintomas, levando ao ganho gradativo de peso (Capitão e Tello, 2004). Porém existem divergências entre autores, alguns relatam que há uma probabilidade de até $25 \%$ de obesos apresentarem os transtornos (Simon et al, 2006) e outros não encontram evidências (FABRICATORE; WADDEN, 2003).

Tanto a obesidade quanto os transtornos alimentares, são considerados patologias, que nos últimos vinte anos aumentaram consideravelmente. Mattos (2007) acredita que esses distúrbios "são manifestações clínicas de Transtornos Narcísicos com perspectivas psicossomáticas, que se correlacionam com a depressão e o Transtorno da Imagem Corporal.” Os Transtornos Alimentares podem se manifestar dentro de intensidades diferentes, mas sempre havendo uma relação entre a perda ou ganho de peso e os obstáculos emocionais.

\subsection{Sintomatologia e qualidade de vida}


Até o início do século XX, o indivíduo obeso era visto como culpado de sua condição (Fischer et al, 1995). Sua incapacidade de autocontrole com relação a comida, construía junto a sociedade uma imagem de limitações e excessos (Wanderley e Ferreira, 2007). Entre tantos prejuízos à saúde, os que advém das exigências relacionadas a padrões corporais são os que mais impactam sob as condições psicológicas, entende-se que não é necessária uma psicopatologia para se tornar obeso, mas a obesidade pode causar uma psicopatologia (SEGAL; FANDINÕ, 2002).

A depressão e a ansiedade são transtornos presentes nos candidatos a cirurgia bariátrica, o que exige cuidados no pré e no pós-operatório. Tal condição leva o indivíduo a comer compulsivamente para aliviar os sintomas (CAPITÃO; TELLO, 2004).

Os transtornos de ansiedade, segundo DSM-V (2014) se caracterizam pelo medo como a resposta emocional a algo que pode ser uma ameaça real ou percebida. O indivíduo com ansiedade antecipa uma provável ameaça, apresentando reações fisiológicas, se colocando em uma posição de luta ou fuga e com o tempo passa a evitar situação que o levem a sentir medo.

Com relação a depressão, o DSM -V (2014) a denomina como Transtorno Depressivo Maior, em que o indivíduo sente desesperança, falta de prazer nas atividades, alterações no sono, apetite e atenção. Diante de um corpo que não se enquadra em padrões e com inúmeras dificuldades de alcançá-lo o indivíduo obeso é suscetível aos sintomas de depressão.

A cirurgia bariátrica é uma opção eficaz e rápida para a perda de peso, o que implica em mudanças corporais radicais em pouco tempo (Franques, 2003). Fato que exige do paciente mudanças psicológicas, o que muitas vezes ocorrem em ritmos diferentes, exigindo uma reestruturação perceptiva diante do novo corpo que está se constituindo (Pruzinsky e Edgerdon, 1990; Tavares, 2003). Tais mudanças implicam em percepções e emoções que podem gerar mais ansiedade e diante das expectativas não alcançadas até mesmo depressão.

Qualidade de vida segundo a Organização Mundial da Saúde (OMS) reflete a percepção do indivíduo sobre suas necessidades, implicando na satisfação, nas oportunidades de alcançar a felicidade e na autorrealização, diante de condições socioeconômicas. Sendo um constructo multidimensional e subjetivo, o que torna sua conceituação difícil. Qualidade de vida vai além de se estar saudável, etimologicamente qualidade deriva de "qualis" que é o modo de ser, com relação a si ou ao grupo, podendo ser positivo ou negativo (SANTINI,2002). 
O termo normalmente remete a saúde e a vivências positivas, contudo devido a sua complexidade implica na percepção do indivíduo sobre sua posição na sociedade, seus objetivos, preocupações e expectativas. Considerando, o estado de saúde, lazer, hábitos e estilo de vida (PUCCI et al, 2012).

No contexto desenvolvido nesta pesquisa, cabe lembrar o quanto a obesidade impacta na saúde, na longevidade e na qualidade de vida de um indivíduo. Promovendo limitações físicas, alterações de humor e da autoestima. Além das comorbidades, o indivíduo obeso tem enorme dificuldade em realizar tarefas diárias, inclusive no trabalho. Fator que implicará no sustento, nas vivências sociais e na realização pessoal (Mariano, Monteiro e Paula, 2013). Com o intuito de aumentar a qualidade de vida, a opção da cirurgia bariátrica possibilita melhora na locomoção, redução dos prejuízos psicossociais e doenças crônicas (MELO; MANCINI, 2009; LEAL; BALDIN, 2007).

\subsection{Cirurgia bariátrica}

Segal e Fandiño (2002) colocam que a obesidade é uma doença de origem multifatorial, desde genéticos até socioambientais. A obesidade mórbida implica em inúmeros distúrbios, como cardiovasculares, ortopédicos, digestivos e respiratórios (Garrido Jr., 2002). Diante deste contexto, o incentivo a prevenção e aos tratamentos a obesidade aumentaram. No Brasil, pesquisas realizadas pelo Instituto Brasileiro de Geografia e Estatística (IBGE) e Ministério da Saúde, estimam um aumento do número de casos de sobrepeso e obesidade, aproximadamente 65 milhões de pessoas estão com sobrepeso, 14 milhões com obesidade e 4 milhões com obesidade mórbida (Berti e Caravatto, 2012). A cirurgia bariátrica foi adotada como um procedimento eficaz em vários países e teve início no Brasil na década de 1970 (Diniz e Maciante, 2012), até então, a dificuldade no tratamento à obesidade mórbida era na manutenção da perda de peso. O procedimento possibilita minimizar as falhas nos tratamentos clínicos e nutricionais (MAGDALENO JR.; CHAIM; TURATO, 2009).

Conhecida também como gastroplastia ou cirurgia de redução de estômago, a cirurgia bariátrica é um método adotado por quem não consegue perder peso pelos métodos tradicionais (Repetto, 2003), apresentando um índice de massa corporal (IMC) acima de 40 $\mathrm{Kg} / \mathrm{m}^{2}$ ou acima de $35 \mathrm{Kg} / \mathrm{m}^{2}$ com a presença de doenças comórbidas, e não apresentar disfunções endócrinas como Síndrome de Cushing e realizar uma avaliação psicológica 
favorável para administrar as mudanças que virão com o procedimento (GARRIDO JR. et al, 2002).

Segundo a Sociedade Brasileira de Cirurgia Bariátrica e Metabólica (2017), a cirurgia bariátrica pode ser disabsortiva, restritiva ou mista. A técnica restritiva limita o volume de sólidos que o paciente possa vir a ingerir, dentro desta técnica está a Gastroplastia Vertical Bandada, que usa um anel limitando o reservatório gástrico; a Gastroplastia Vertical, que fecha parte do estômago através de uma sutura e a Banda Gástrica ajustável, que usa um dispositivo subcutâneo para regular o volume do reservatório gástrico. A técnica disabsortiva permite o paciente comer, porém limita a absorção de nutrientes, através de uma redução do intestino delgado, a Cirurgia de Payne, reduz o caminho que a comida irá percorrer. Com relação a técnica mista, o Bypass Gástrico Y de Roux, restringe tanto a quantidade de alimento quanto os nutrientes.

O procedimento adotado proporcionará uma redução de peso de até $40 \%$ em um ano a partir da cirurgia, o estômago é reduzido em média 20 centímetros cúbicos, perdendo $90 \%$ da capacidade de absorção (Segal e Fandiño, 2002). Dessa forma, o estômago não suporta a quantidade habitual de alimento, transmitindo para o cérebro uma mensagem de saciedade possibilitando que a pessoa consuma bem menos que antes (Marcelino e Patrício, 2011). O objetivo da cirurgia bariátrica é melhorar a qualidade e o tempo de vida, resolvendo os problemas físicos e psicossociais causados pela obesidade (COSTA et al 2009).

No período pré-operatório, o paciente passa por avaliações físicas e psicológicas, sendo informado das alterações que passará, estando ciente da dimensão do processo. $\mathrm{O}$ procedimento é contraindicado quando o paciente não se vê preparado para o pós-operatório ou quando há a presença de um transtorno psiquiátrico incapacitando sua percepção (Segal e Fandiño, 2002). O indivíduo obeso, apresenta um sofrimento psíquico gerado pelo preconceito social (Coutinho,1999), ou pelo surgimento de sintomas depressivos ou ansiosos. Segundo o Conselho Federal de Psicologia (2013) o psicólogo e/ou psiquiatra que integra a equipe multidisciplinar deve se atentar nesta fase do processo, para o uso de substâncias psicoativas, ou para os quadros psicóticos ou demenciais. Inclusive certificar que o paciente apresenta capacidade cognitiva para entender as demandas pós-operatórias.

Flores (2014), cita os fatores psicossociais que determinam a qualificação do paciente à cirurgia bariátrica: compreensão do paciente quanto ao procedimento e as mudanças futuras, expectativas quanto aos resultados, habilidade de adaptação pós-operatório, comportamento 
alimentar, comorbidades psiquiátricas, motivos para se candidatar à cirurgia, suporte da rede de apoio, uso de substâncias, autoestima, qualidade de vida ,ideação suicida e histórico de possíveis traumas. Alguns problemas que impedem, frequentemente o processo de avaliação é o uso de substâncias lícitas ou ilícitas, transtornos alimentares, transtornos psicóticos, de humor e tentativas de suicídio. Quanto a compulsão alimentar, o autor afirma em sua pesquisa que os autores se dividem, enquanto alguns acreditam que o transtorno pode entrar em remissão após a cirurgia, outros acreditam que o paciente pode voltar a ter este comportamento, não sendo uma contraindicação, contudo que seja considerada a possibilidade de normalizar tal comportamento através de terapia antes do procedimento.

O período pós-cirúrgico caracteriza-se pelas mudanças, alimentares, sociais e comportamentais. O que exigirá do paciente adaptação e adesão aos novos hábitos, a fim de não comprometer os resultados da cirurgia. O paciente é monitorado pela equipe multidisciplinar (Fandiño et al, 2004), pois há incidência de transtornos como o de ansiedade, humor, alimentares e comportamentos de risco (Leal e Baldin, 2007). Oliveira (2006) ressalta as três fases pós-cirúrgicas em que o paciente passa, na primeira fase, que dura aproximadamente três meses, o paciente vai enfrentar a realidade, mesmo perdendo peso rapidamente, diante dos conflitos perceberá que nem todos os seus problemas estavam vinculados ao sobrepeso. Na segunda fase, que ocorre entre o sexto e oitavo mês, $30 \%$ do peso já foi eliminado e uma nova imagem começa a ser notada por todos, promovendo momentos de euforia. E na terceira fase, quando um ano pós-cirurgia se completa, o peso se estabilizou, contudo nem todos os objetivos foram alcançados, inclusive a própria aceitação da nova imagem corporal. Sendo necessário um maior apoio psicoterápico para trabalhar os aspectos de uma nova identidade.

As alterações nos contornos corporais nem sempre são percebidas havendo uma certa distorção nos primeiros meses. A insatisfação diante dos resultados, como a flacidez da pele em várias regiões do corpo, pode levar o paciente a adotar comportamentos de risco, através da má alimentação, exercícios em excesso, gerando uma carência nutricional e ao desenvolvimento de transtornos alimentares e do humor (REZENDE, 2011; BRANCO; HILÁRIO; CINTRA, 2006).

A obesidade é uma doença crônica de origem multifatorial, que compromete a qualidade e a expectativa de vida. Possibilita o surgimento de outras doenças e expõe o sujeito a fatores psicossociais. Dependendo do grau de obesidade, que é medido pelo Índice de Massa 
Corporal (IMC) a adesão a dietas convencionais e a hábitos saudáveis é algo impossível, devido à falta de motivação e a demora em se obter resultados. A cirurgia bariátrica surge como uma solução eficaz, que traz resultados rápidos, contudo exige acompanhamento pré e pós o procedimento de uma equipe multidisciplinar, bem como a dedicação do paciente em se adaptar à nova vida.

O objetivo geral desta pesquisa é avaliar as repercussões da cirurgia bariátrica nos sintomas de ansiedade, depressão e na qualidade de vida, buscando conceituar obesidade e identificar seus prejuízos. Investigar a relação da obesidade com os sintomas de depressão, ansiedade e qualidade de vida. Relatar os possíveis benefícios da cirurgia bariátrica.

\section{METODOLOGIA}

A busca bibliográfica foi realizada nas seguintes bases eletrônicas: Google Acadêmico, SCielo, PubMed/Medline, entre outros acessados via Biblioteca Virtual de Saúde (BVS). Além disto, artigos relevantes para a revisão serão utilizados nas referências dos estudos que fecharam critérios para a inclusão na revisão.

Os "termos chave" da busca foram: (1) cirurgia bariátrica; (2) depressão; (3) ansiedade e (4) qualidade de vida. Criou-se uma lista de descritores para cada termo e a busca final foi realizada com a intersecção entre as quatro listas.

Dentre os artigos selecionados, a presente revisão tratou apenas daqueles cujo foco relacionou cirurgia bariátrica, depressão, ansiedade e qualidade de vida.

\section{RESULTADOS}

Foram encontrados 36 artigos científicos na base de dados Biblioteca Virtual em Saúde (BVS), 33 Medline e 3 Lilacs. A busca realizada se ateve aos últimos dez anos e foi realizada com o seguinte cruzamento: bariatric surgery, depression, anxiety e life quality. Com base na análise dos resumos, foram selecionados somente estudos que atenderam aos seguintes critérios de inclusão: (1) foram publicados em forma de artigo científico; (2) investigaram pacientes pós cirurgia bariátrica; (3) pesquisaram sintomas de depressão, ansiedade e fatores relacionados a qualidade de vida e (4) os participantes das pesquisas eram 
seres humanos. Foram excluídos da análise os estudos que investigaram (1) somente candidatos à cirurgia bariátrica e (2) pacientes adolescentes.

O rastreio foi realizado durante os meses de agosto e setembro de 2019, sendo encontrado um artigo repetido (Tae, et al, 2014). Por fim, uma análise dos artigos que atenderam os critérios de inclusão e exclusão foi realizada, com a intenção de definir o número final de estudos a serem revisados. Sendo assim, a amostra deste estudo resultou em 8 artigos, que atenderam as variáveis de interesse, mais duas revisões bibliográficas (Olguin, et al, 2014; Ghoneim e O'hara, 2016) que serão usadas na discussão desta pesquisa. As principais informações dos estudos incluídos na presente revisão estão disponibilizadas na Tabela 1.

Tabela 1 - Informações acerca dos estudos incluídos na revisão de literatura

(continua)

\begin{tabular}{|c|c|c|c|c|}
\hline Autores / Ano & Amostra & Delineamento & Medidas & $\begin{array}{l}\text { Principais resultados/ } \\
\text { Conclusão }\end{array}$ \\
\hline $\begin{array}{l}\text { Burgmer et al } \\
\quad[2014]\end{array}$ & $\begin{array}{l}148 \text { pacientes ( } 47 \\
\text { homens, } 101 \\
\text { mulheres) com } \\
\text { idade média de } 38 \\
\text { anos. }\end{array}$ & $\begin{array}{l}\text { Os pacientes foram } \\
\text { contatados um ano, } 2 \\
\text { anos e } 4 \text { anos após a } \\
\text { cirurgia. Foram } \\
\text { avaliados o curso do } \\
\text { peso, a mudança na } \\
\text { depressão, ansiedade } \\
\text { e autoestima. }\end{array}$ & $\begin{array}{c}\text { Os sintomas } \\
\text { depressivos e de } \\
\text { ansiedade foram } \\
\text { avaliados com o } \\
\text { Hospital Anxiety } \\
\text { and depression } \\
\text { scale (HADS-D). A } \\
\text { autoestima foi } \\
\text { avaliada com a } \\
\text { Escala de } \\
\text { autoestima de } \\
\text { Rosenberg. }\end{array}$ & $\begin{array}{l}\text { Houve uma considerável } \\
\text { perda de peso e melhoria } \\
\text { em vários aspectos } \\
\text { psicológicos no primeiro } \\
\text { ano após o } \\
\text { procedimento. }\end{array}$ \\
\hline $\begin{array}{l}\text { Chalut-Carpen } \\
\text { tier et al } \\
(2014)\end{array}$ & $\begin{array}{l}38 \text { pacientes } \\
\text { investigados antes } \\
\text { da cirurgia } \\
\text { bariátrica e } \\
\text { somente } 27 \text { deles } \\
\text { reexaminados } 6 \\
\text { meses após o } \\
\text { procedimento. }\end{array}$ & $\begin{array}{l}\text { Estudo prospectivo } \\
\text { de coorte. }\end{array}$ & $\begin{array}{l}\text { Ansiedade e } \\
\text { depressão foram } \\
\text { medidas pela escala } \\
\text { HAD. Qualidade de } \\
\text { vida foi avaliada } \\
\text { com o instrumento } \\
\text { WQOLLITE. }\end{array}$ & $\begin{array}{l}\text { O IMC foi drasticamente } \\
\text { reduzido. Não foram } \\
\text { observadas modificações } \\
\text { nos parâmetros de } \\
\text { ansiedade e depressão. } \\
\text { Quanto a qualidade de } \\
\text { vida, melhora } \\
\text { significativa, exceto no } \\
\text { domínio sexual. }\end{array}$ \\
\hline
\end{tabular}


Tabela 1 - Informações acerca dos estudos incluídos na revisão de literatura

(conclusão)

\begin{tabular}{|c|c|c|c|c|}
\hline Autores / Ano & Amostra & Delineamento & Medidas & $\begin{array}{l}\text { Principais resultados/ } \\
\text { Conclusão }\end{array}$ \\
\hline $\begin{array}{l}\text { Herpertz et al } \\
\quad(2015)\end{array}$ & $\begin{array}{c}152 \text { pacientes } \\
\text { submetidos à } \\
\text { cirurgia bariátrica; } \\
249 \text { pacientes com } \\
\text { tratamento de perda } \\
\text { de peso } \\
\text { convencional e } 128 \\
\text { pacientes do grupo } \\
\text { controle. } \\
\text { Depois de } 9 \text { anos } \\
55 \% \text { pacientes pós } \\
\text { CB; } \\
51 \% \text { pacientes } \\
\text { perda de peso } \\
\text { convencional; } \\
65 \% \text { pacientes s/ } \\
\text { tratamento de perda } \\
\text { de peso. }\end{array}$ & $\begin{array}{l}\text { Coorte longitudinal } \\
\text { prospectivo. }\end{array}$ & $\begin{array}{c}\text { Ansiedade e } \\
\text { depressão - } \\
\text { Hospital Anxiety } \\
\text { and depression scal } \\
\text { (HADS); } \\
\text { Aspectos mentais- } \\
\text { Short - form } \\
\text { (SF-36); } \\
\text { Autoestima - } \\
\text { escala de } \\
\text { autoestima } \\
\text { Rosenberg (RSES). }\end{array}$ & $\begin{array}{l}\text { Pacientes pós cirurgia } \\
\text { experimentaram } \\
\text { melhorias iniciais em } \\
\text { depressão, ansiedade, } \\
\text { autoestima. Essas } \\
\text { melhorias de } \\
\text { deterioraram no decorrer } \\
\text { da avaliação de } 9 \text { anos, } \\
\text { identificadas iguais ou } \\
\text { piores aos resultados pré } \\
\text { CB. }\end{array}$ \\
\hline $\begin{array}{l}\text { Matini et al } \\
\text { [2014] }\end{array}$ & $\begin{array}{l}67 \text { candidatos com } \\
\text { obesidade } \\
\text { e mórbida. Idade } \\
\text { entre } 36 \text { a } 86 \text { anos, } \\
\text { com IMC médio de } \\
48,8 \mathrm{Kg} / \mathrm{m}^{2}\end{array}$ & $\begin{array}{l}\text { Estudo observacional } \\
\text { prospectivo, com } \\
\text { coleta de dados } \\
\text { através de } \\
\text { instrumentos } \\
\text { conduzido por } \\
\text { psiquiatras. }\end{array}$ & $\begin{array}{l}\text { Questionário Short } \\
\text { form health survery } \\
\text { para qualidade de } \\
\text { vida. Entrevista } \\
\text { clínica estruturada } \\
\text { para transtornos } \\
\text { DSM-IV e EDI-3 } \\
\text { para identificar } \\
\text { transtornos } \\
\text { alimentares. }\end{array}$ & $\begin{array}{c}\text { Após } 6 \text { meses, a média } \\
\text { de perda de peso foi de } \\
29,3 \mathrm{Kg} \text { e o IMC reduziu } \\
\text { para } 35,7 \mathrm{Kg} / \mathrm{m}^{2} \text {. Quanto } \\
\text { a depressão e ansiedade } \\
\text { não houve uma diferença } \\
\text { significativa. } \\
\text { Após a cirurgia bariátrica } \\
\text { houve perda de peso, } \\
\text { porém não houve } \\
\text { alteração significativa } \\
\text { nos quadros de depressão } \\
\text { e ansiedade. }\end{array}$ \\
\hline $\begin{array}{l}\text { Rutledge et al } \\
\text { [2012] }\end{array}$ & $\begin{array}{c}55 \text { pacientes } \\
\text { submetidos a CB há } \\
5 \text { anos (1999- } \\
\text { 2007). }\end{array}$ & $\begin{array}{l}\text { Os métodos de estudo } \\
\text { incluíram } \\
\text { componentes } \\
\text { retrospectivos e } \\
\text { prospectivos. }\end{array}$ & $\begin{array}{l}\text { Uso do histórico } \\
\text { médico e } \\
\text { avaliações } \\
\text { pré-operatórios. } \\
\text { Também foram } \\
\text { analisados os } \\
\text { exames realizados } \\
\text { após o } \\
\text { procedimento. }\end{array}$ & $\begin{array}{l}\text { Evidências mostraram a } \\
\text { redução do uso de } \\
\text { antidepressivos e terapia } \\
\text { voltada ao transtorno, } \\
\text { porém houve aumento no } \\
\text { uso de ansiolítico e } \\
\text { terapia para ansiedade. } \\
\text { Apesar das melhorias } \\
\text { metabólicas há a } \\
\text { necessidade de uma } \\
\text { atenção continua à saúde } \\
\text { mental. }\end{array}$ \\
\hline $\begin{array}{l}\text { Sierzatowicz. } \\
\text { et al [2017] }\end{array}$ & $\begin{array}{c}57 \text { mulheres e } 43 \\
\text { homens com idade } \\
\text { média de } 40 \text { anos, } \\
\text { com IMC de } 36 \text { a } \\
40 \mathrm{Kg} / \mathrm{m}^{2} . \text { Os } \\
\text { pacientes foram }\end{array}$ & $\begin{array}{l}\text { Estudo prospectivo } \\
\text { de coorte. }\end{array}$ & $\begin{array}{c}\text { A Análise } \\
\text { Bariátrica e } \\
\text { Relatório Resultado } \\
\text { System (BAROS) } \\
\text { foi utilizado para }\end{array}$ & $\begin{array}{l}\text { Seis meses e } 1 \text { ano após } \\
\text { a cirurgia bariátrica, o } \\
\text { IMC }>40 \text { caiu de } 69 \% \\
\text { para } 14 \% \text {. Com relação a } \\
\text { escala de Autoavaliação }\end{array}$ \\
\hline
\end{tabular}




\begin{tabular}{|l|c|c|c|c|}
\hline & $\begin{array}{c}\text { submetidos a } \\
\text { diferentes }\end{array}$ & $\begin{array}{c}\text { avaliar a qualidade } \\
\text { de vida. }\end{array}$ & $\begin{array}{c}\text { de Depressão e } \\
\text { Ansiedade, }\end{array}$ \\
\hline
\end{tabular}

(conclusão)

\begin{tabular}{|c|c|c|c|c|}
\hline Autores / Ano & Amostra & Delineamento & Medidas & $\begin{array}{l}\text { Principais resultados/ } \\
\text { Conclusão }\end{array}$ \\
\hline & técnicas de CB. & & $\begin{array}{l}\text { A gravidade dos } \\
\text { transtornos do } \\
\text { humor (depressão, } \\
\text { ansiedade) foi } \\
\text { avaliada utilizando } \\
\text { a Escala de } \\
\text { Autoavaliação da } \\
\text { depressão e } \\
\text { ansiedade de } \\
\text { Kokoszka. }\end{array}$ & $\begin{array}{c}\text { verificou-se } 48 \% \text { dos } \\
\text { participantes apresentava } \\
\text { gravidade média da } \\
\text { depressão e ansiedade. } \\
\text { O tipo de CB pode } \\
\text { determinar sua eficácia. } \\
\text { Isso inclui } \\
\text { melhora da saúde, } \\
\text { controle de } \\
\text { comorbidades, redução } \\
\text { de problemas } \\
\text { psicológicos e aumento } \\
\text { da QV. }\end{array}$ \\
\hline $\begin{array}{l}\text { Sockalingan et } \\
\text { al (2014). }\end{array}$ & $\begin{array}{l}164 \text { pacientes que } \\
\text { se submeteram a } \\
\text { CB entre } 2010 \text { a } \\
\text { 2012. Avaliados no } \\
\text { pré-operatório e } 12 \\
\text { meses após a } \\
\text { cirurgia. }\end{array}$ & $\begin{array}{l}\text { Estudo prospectivo } \\
\text { de Coorte. }\end{array}$ & $\begin{array}{l}\text { LEAPS para avaliar } \\
\text { produtividade } \\
\text { laboral. Sintomas } \\
\text { depressivos } \\
\text { avaliados pelo } \\
\text { PHQ9 e sintomas } \\
\text { de ansiedade } \\
\text { GAD7. QV medido } \\
\text { pelo SF-36. O } \\
\text { MINI foi o } \\
\text { instrumento usado } \\
\text { identificar } \\
\text { patologias } \\
\text { psiquiátricas. }\end{array}$ & $\begin{array}{l}\text { Os resultados concluíram } \\
\text { que CB proporciona uma } \\
\text { melhora no que diz } \\
\text { respeito a produtividade. } \\
\text { Contudo os autores do } \\
\text { estudo sugerem que } \\
\text { intervenções destinadas a } \\
\text { apoiar e manter a } \\
\text { melhoria da saúde } \\
\text { mental após a CB, } \\
\text { possam potencializar os } \\
\text { resultados. Havendo a } \\
\text { necessidade de novos } \\
\text { estudos para além dos } 12 \\
\text { meses. }\end{array}$ \\
\hline $\begin{array}{l}\text { Tae et al } \\
{[2014]}\end{array}$ & $\begin{array}{l}32 \text { mulheres, com } \\
\text { média de idade de } \\
41 \text { anos, maioria } \\
\text { casada, sem } \\
\text { comorbidades, } \\
\text { brancas, católicas e } \\
\text { com ensino médio } \\
\text { completo. }\end{array}$ & $\begin{array}{l}\text { Estudo longitudinal } \\
\text { prospectivo. As } \\
\text { avaliações foram } \\
\text { aplicadas } 3 \text { meses } \\
\text { antes da cirurgia e } \\
\text { entre } 6 \text { a } 10 \text { meses } \\
\text { após a cirurgia. }\end{array}$ & $\begin{array}{c}\text { Instrumentos } \\
\text { usados Inventário } \\
\text { de Depressão de } \\
\text { Beck, Inventário de } \\
\text { ansiedade } \\
\text { traço-estado } \\
\text { (IDATE-T), } \\
\text { Edinburgh (BITE), } \\
\text { Escala de qualidade } \\
\text { de vida } \\
\text { (WHOQOL) e } \\
\text { Inventário sobre } \\
\text { uso de psicoativas } \\
\text { substâncias. }\end{array}$ & $\begin{array}{c}\text { Houve redução nos } \\
\text { sintomas depressivos e } \\
\text { ansiosos. Aumento } \\
\text { significativo na } \\
\text { qualidade de vida nos } \\
\text { domínios físico, psíquico } \\
\text { e ambiental. Seis meses } \\
\text { após a CB, foi observada } \\
\text { a diminuição do uso de } \\
\text { antidepressivos e } \\
\text { moderadores de apetite. } \\
\text { Concluiu-se que houve } \\
\text { diminuição de } \\
\text { sintomatologia. } \\
\text { Psiquiátrica (depressão, } \\
\text { ansiedade, bulimia). } \\
\text { Redução do uso do uso } \\
\text { de substâncias } \\
\text { psicoativas e melhora da } \\
\text { qualidade de vida. }\end{array}$ \\
\hline
\end{tabular}

Fonte: Autoria própria, 2019 
No que tange às características dos participantes, verificou-se que a maioria investigou o impacto da redução do peso nas patologias psiquiátricas, tendo um estudo realizado somente com sujeitos idosos (Rutledge et al, 2012) e o outro somente com mulheres (Tae et al, 2014). Além disso, os estudos variaram o período de análise entre 6 meses a 9 anos após a cirurgia.

Quanto ao número de participantes o conjunto dos 8 estudos selecionados reuniu uma amostra de 1.133 pacientes, sendo o número mínimo de 32 (Tae et al, 2014) e o número máximo de 529 (Herpertz et al, 2015). No que se refere ao sexo dos participantes, todos os estudos incluíram sujeitos do sexo feminino. Os instrumentos utilizados para mensurar depressão, ansiedade, e qualidade de vida foram definidos conforme o país em que o estudo foi desenvolvido.

Entre os artigos selecionados para esta revisão, o mais antigo foi realizado por Rutledge e colaboradores em 2012. Desenvolvido em San Diego, nos EUA, a análise ocorreu a partir dos registros médicos do sistema de saúde local de pacientes que passaram pela cirurgia bariátrica entre 1999 e 2007. A amostra de 55 pacientes, tinha na sua maioria mulheres com idade média de 65 anos e IMC médio de $35 \mathrm{Kg} / \mathrm{m}^{2}$. O estudo constatou que houve declínio nas taxas de uso de antidepressivos e terapia, porém as taxas de uso de ansiolíticos e terapia para a ansiedade se mantiveram. Não havendo consistência nos resultados, que dizem respeito as melhorias nos transtornos de depressão e ansiedade após 5 anos da cirurgia bariátrica, concluiu-se a necessidade de uma atenção continua à saúde mental no processo após a cirurgia bariátrica. Considerando o tempo pós cirurgia bariátrica, Burgmer et al ( 2014) em seu estudo com 148 pacientes, durante os 4 anos após o procedimento, obtiveram resultados que mostraram uma considerável perda de peso e melhoria em vários aspectos psicológicos no primeiro ano após a cirurgia bariátrica, no entanto nos anos posteriores não foram encontradas evidências de tais melhorias. O retorno dos sintomas, como salientaram os autores, levaram a reflexão que o paciente se adapta a vida diária e a perda de peso se torna mais lenta, havendo uma redução do entusiasmo inicial e os problemas não vinculados a obesidade voltam a ter maior relevância. Resultados apoiados pelo estudo de Herpertz et al (2015), que teve uma amostra inicial de 152 pacientes submetidos a cirurgia bariátrica, 249 em tratamento convencional para perda de peso e 128 sem tratamento para emagrecimento, acompanhados por 9 anos. Concluindo que a cirurgia bariátrica é um procedimento eficaz e colabora com aspectos físicos, porém a melhoria da saúde mental volta ao estágio pré-operatório ou piora ao longo do tempo. 
Com o objetivo de avaliar o efeito do IMC sobre a qualidade de vida e os níveis de depressão, Sierzantowicz et al (2017) reuniu 57 mulheres e 43 homens, com idade média de 40 anos submetidos a diferentes tipos de cirurgia bariátrica no período de 6 meses a um ano após o procedimento. Comprovando que o tempo pós cirúrgico pode ter maior impacto na qualidade de vida, independentemente do tipo de procedimento, porém a escolha do tipo de cirurgia bariátrica pode influenciar na eficácia, que a longo prazo refletirá na melhora da saúde, controle de comorbidades, redução de problemas psicológicos e aumento da qualidade de vida.

Os estudos realizados analisando os resultados a curto prazo (Matini et al, 2014; Sierzatowicz et al 2017; Tae, et al, 2014, Chalut-Carpentier, 2014 e Sockalingan et al, 2014), ou seja, entre 6 a 12 meses após a cirurgia bariátrica mostraram que a redução de peso foi significativa, não apresentando alterações nos sintomas de depressão e ansiedade ( Matini et al, 2014 e Chalut-Carpentier et al, 2014), ou havendo somente uma redução nos sintomas (Tae, et al, 2014), ou ainda uma expectativa de melhora a longo prazo (Sierzatowicz et al, 2017). Quanto ao constructo qualidade de vida, de modo geral, houve um aumento, com exceção na pesquisa de Chalut-Carpentier et al (2014) que mostrou uma queda no domínio que inclui a vida sexual. E na pesquisa de Sockalingam et al (2014), com 164 pacientes avaliados 12 meses após a cirurgia, mesmo constatando resultados positivos no domínio que inclui produtividade laboral, os autores advertem a necessidade de intervenções psicológicas para a melhoria dos resultados e que estudos sejam realizados para além dos 12 meses.

\section{DISCUSSÃO}

A presente revisão teve como objetivo avaliar as repercussões da cirurgia bariátrica nos sintomas de ansiedade, depressão e na qualidade de vida. As buscas nos bancos de dados eletrônicos resultaram na identificação de 8 artigos, estes estudos envolveram uma amostra de 1.133 pacientes, com idade média de 44 anos, sendo 332 homens e 801 mulheres.

Considerada uma doença crônica, a obesidade toma dimensões preocupantes, por ser fator de risco para inúmeras patologias e tida como um problema de saúde pública em vários países (Magdaleno Jr., Chaim e Turato, 2009). O indivíduo obeso está predisposto ao aparecimento ou agravamento de doenças crônicas e de sofrimentos psicossociais (Marcelino e Patrício, 2011). Para classificar a gravidade do quadro, cabe ao cálculo do Índice de Massa 
Corporal (IMC) indicar o $\mathrm{Kg} / \mathrm{m}^{2}$ que identificará o grau dentro de uma normalidade ou o extremo que é o grau III, que denomina a obesidade como mórbida ou severa.

A obesidade mórbida traz consigo inúmeras complicações que afetam e até colocam em risco a vida do indivíduo. Além dos distúrbios fisiológicos, limitações físicas, as vivências geram frustrações, ansiedade, estresse e depressão. Fatores somados que implicam no comportamento e na qualidade de vida (Marcelino e Patrício, 2011). As repercussões da cirurgia bariátrica sob os sintomas de depressão, ansiedade e qualidade de vida foram demonstrados em todos os estudos selecionados.

O constructo qualidade de vida remete ao conceito de saúde plena, mas por ser de difícil compreensão devido a sua complexidade e depender da percepção que o indivíduo tem de sua própria vida, dentro do contexto sociocultural e frente aos seus valores, expectativas, padrões e preocupações. Existindo, então, a necessidade de instrumentos que meçam e avaliem amplamente, levando em conta seus diversos domínios: físico, psicológico, nível de independência, relações sociais, ambiente e aspectos espiritualidade/religião (Pereira, Teixeira e Santos, 2012). Nos estudos selecionados instrumentos semelhantes foram usados, apresentando um formato de questionário separando os domínios que avaliam a percepção do paciente sob sua qualidade de vida.

Os estudos mostraram (Tae et al, 2014 ; Matini et al, 2014; Chalut-Carpentier et al, 2014; Sockalingan et al, 2014)de maneira geral uma melhora na qualidade de vida, no período entre 6 a 12 meses após a cirurgia bariátrica. Porém, tais resultados não se mostraram consistentes em todos os domínios, como no domínio social (Tae et al, 2014), sexual (Chalut-Carpentier et al, 2014) e mental (Matini et al, 2014). Com relação ao domínio independência, que inclui a produtividade o estudo de Sockalingan et al (2014) concluiu que houve uma melhora significativa na área laboral, contudo, intervenções destinadas a melhoria da saúde mental após a cirurgia bariátrica possam potencializar os resultados, havendo também a necessidade de pesquisas além dos 12 meses do procedimento.

A cirurgia bariátrica possibilita a perda rápida de peso, permitindo a diminuição das comorbidades médicas e psiquiátricas, com o intuito da melhoria da qualidade de vida (Olguin, Carvajal e Fuentes, 2014). Habitualmente, as pessoas que procuram tratamento para a obesidade, seja cirúrgico ou farmacológico, possuem um histórico de depressão e ansiedade. O indivíduo obeso é mais vulnerável à depressão, os fatores responsáveis a esta 
suscetibilidade não são claros, podendo contribuir para isso a estigmatização e a angústia emocional gerada pelas complicações da própria obesidade (GHONEIM; O’HARA, 2016).

A prevalência de uma maior procura pela cirurgia bariátrica por mulheres foi mostrada em todos os estudos relacionados, inclusive havendo um estudo somente com mulheres (Tae et al, 2014). Oliveira, Mereghi e Jesus (2014), afirmam em sua pesquisa que a mulher obesa busca a cirurgia bariátrica devido suas vivências e principalmente pelas exigências sociais. Constatam que a construção da obesidade se dá pelos hábitos alimentares inadequados no decorrer da vida.

Nos estudos que observaram pacientes no período de 2 a 9 anos após a cirurgia bariátrica (Burgmer et al, 2014; Rutledge et al, 2012; Herpertz et al, 2015) observou-se uma melhoria nos aspectos psicológicos no primeiro ano (Burgmer et al,2014). Entretanto, um dos estudos (Rutledge et al, 2012) realizado com idosos, após 5 anos da cirurgia bariátrica, apresentou um aumento do uso de ansiolítico e terapia para ansiedade. A deterioração na melhora do humor foi observada na pesquisa realizada por 9 anos (Herpertz et al, 2015) com 152 pacientes, em que a partir dos 4 anos pós cirurgia bariátrica sinais de decepção com os resultados do procedimento refletiram nos comportamentos alimentares, somados às sequelas médicas e o medo de reganho de peso. A perda de peso que a cirurgia bariátrica promove, em um primeiro momento viabiliza a aceitação social, promove mudanças de estilo de vida e da própria aceitação corporal, fatores que podem criar expectativas irreais (Olguin, Carvajal e Fuentes, 2014). Assim a perda do entusiasmo inicial, a redução de peso mais lenta, a estagnação ou até mesmo o reganho de peso podem levar o paciente a quadros de ansiedade e o declínio de certos aspectos da qualidade de vida. A motivação inicial passa a dar espaço a vida cotidiana não restrita às transformações pós cirúrgicas, havendo a necessidade de apoio psicológico para que a cirurgia bariátrica tenha sucesso a longo prazo (BURGMER et al, 2014).

\section{CONSIDERAÇÕES FINAIS}

A cirurgia bariátrica é atualmente uma opção terapêutica para solucionar a obesidade mórbida e as doenças comórbidas. Compreende-se como obesidade o excesso de gordura que se origina por inúmeros fatores que acontecem de forma isolada ou combinada, que podem ser de origem fisiológica, social ou psicológica entre outras. Frente a uma tendência na população 
ao desenvolvimento da obesidade, cabe ao Índice de Massa Corporal (IMC) estabelecer, através de um cálculo de divisão que envolve peso e o dobro da altura, e seu produto indicará uma normalidade ou uma severidade.

A obesidade não é considerada uma doença psiquiátrica, contudo é um fator de risco para o desenvolvimento de transtornos de humor e alimentares. Coube a esta pesquisa investigar a relação da obesidade com os transtornos de ansiedade, depressão e qualidade de vida e avaliar as repercussões da cirurgia bariátrica sob estes aspectos. A maioria dos artigos selecionados para este estudo, mostraram melhoras nos quadros de depressão, ansiedade e um aumento na qualidade de vida, principalmente nos primeiros 12 meses após o procedimento.

No que diz respeito à amostra dos estudos, foi observado uma maior incidência de indivíduos do sexo feminino. Esses além da busca pela cirurgia bariátrica para redução de peso, também buscam uma aceitação social. O que a princípio gera situações ansiogênicas, comprometendo a qualidade de vida e a saúde mental dessas mulheres. Tema que poderá ser pesquisado futuramente.

Quanto à limitação deste trabalho, salienta-se a dificuldade para identificar estudos que avaliassem o pós-operatório, além dos 12 meses da cirurgia bariátrica. Os poucos artigos encontrados constataram que mesmo com a perda de peso significativa, o indivíduo retorna a ter sintomas de depressão e ansiedade após aproximadamente 24 meses da cirurgia. Tal sintomatologia se dá devido às expectativas e às mudanças exigidas a partir do procedimento, se fazendo necessária uma reorganização do indivíduo.

Os resultados mostraram que somente a cirurgia bariátrica não soluciona a obesidade e suas ressonâncias. Fazendo-se necessária a implantação de estratégias que envolvam o paciente e sua rede social, para que haja uma melhor adesão aos novos hábitos alimentares e rotineiros. A ação interdisciplinar é essencial, e manter um olhar biopsicossocial sob o indivíduo a longo prazo fará com que os sintomas de ansiedade e depressão sejam controlados e consequentemente aumente sua qualidade de vida.

Os futuros estudos deverão considerar suas pesquisas com prognósticos para além dos 12 meses da cirurgia bariátrica, possibilitando uma melhor compreensão dos impactos que esta provoca no indivíduo obeso e suas vivências.

\section{REFERÊNCIAS BIBLIOGRÁFICAS}


ADES, L., KERBAUY, R. R. Obesidade: realidade e indagações. Psicologia USP, 13(1), 197-216. 2002.

ALMEIDA, Sebastião S.; ZANATTA, Daniela P.; REZENDE, Fabiana F. Imagem corporal, ansiedade e depressão em pacientes obesos submetidos à cirurgia bariátrica. Estudos de Psicologia. Natal - RN.2012.

AMERICAN PSYCHIATRIC ASSOCIATION. Manual diagnóstico e estatístico de transtornos mentais: DSM-5. 5 ed. Porto Alegre: Artmed, 2014.

ANJOS, Luiz A. Índice de massa corporal como indicador do estado nutricional de adultos: revisão de literatura. Rev. Saúde Pública, São Paulo, v. 26, n. 6, p. 431 - 436, dez. 1992.

ARASAKI, C. H. (2005 Cirurgia bariátrica para tratamento da obesidade. In A. M Claudino \& M. T. Zanella (Orgs.), Guia de transtornos alimentares e obesidade, p. 287-296. São Paulo: Manole. 2005.

AZEVEDO, A. P., SANTOS, C. C., FONSECA, D.C. Transtorno da compulsão alimentar periódica. Revista de Psiquiatria Clínica, v. 31, 170-172, 2004.

BERTI, L. V., CARAVATTO, P. P. P. Importância da obesidade no Brasil e no mundo. In: M.T.C. Diniz (Org.). Cirurgia bariátrica e metabólica: abordagem multidisciplinar. São Paulo: Atheneu. 2012.

BJÖRNTORP, P., Definition and classification of obesity. In: Fairbuirn e Brownell (eds) Eating disorders and obesity. 2.ed, p. 377 - 381.New York. 2003.

BRANCO, L.M., HILÁRIO, Moe, CINTRA, I.P. Percepção e satisfação corporal em adolescentes e a relação com seu estado nutricional. Revista Psiquiatria Clínica, v. 33, p. $292-296,2006$. 
BURGMER, Ramona; LEGENBAUER, Tanja; MÜLLER, Astrid; ZWAAN, Martina; FISCHER, Charlotte, HERPERTZ, Stephan. Psychological outcome 4 years after restrictive bariatric surgery. Obesity Surgery. Alemanha, v. 24, p. 1670-1678, out., 2014.

CAPITÃO, Cláudio Garcia; TELLO, Renata Raveli. Traço e estado de ansiedade em mulheres obesas. Psicologia Hospitalar, São Paulo, v. 2, n. 2, dez. 2004.

CASH, T.F., PRUZINSKY, T. Body image: a handbook of theory, research, and clinical practive. Nova Iorque: Guilford Press, Nova Iorque, 2002.

CASTRO, C. Ministros da saúde das américas se comprometem a criar políticas públicas para reduzir a obesidade no continente. Associação Brasileira para o Estudo da Obesidade OBESO. São Paulo. 2011. Disponível em: $<$ http://www. abeso.org.br $>$. Acesso em: 13. ago. 2019.

CHALUT-CARPENTIER, Anne; PATAKY, Zoltan; GOLAY, Alain; BOBBIONI-HARSCH, Elisabetta. Involvement of dietary fatty acids in multiple biological and psychological functions, in morbidly obese subjects. Obesity Surgery. Genebra, v.2 5, p. 1031-1038, jun., 2015.

COUTINHO, W. F. O Consenso Latino Americano em Obesidade. Arquivos Brasileiros de Endocrinologia e Metabologia, v. 43, p. 21-67, 1999.

CONSELHO FEDERAL DE MEDICINA. Resolução CFM $\mathbf{n}^{\circ} \mathbf{1 . 7 6 6 / 0 5}$. Brasília, CFM. 2005 .

CONSELHO FEDERAL DE PSICOLOGIA. Sistema de Avaliação de Testes Psicológicos SATEPSI. 2013. Disponível em:< http://www.pol.org.br/satepsi >. Acesso em: 15. set. 2019.

COSTA, Anna Christina C., IVO, Maria L., CANTERO, Wilson B., TOGNINI, João Ricardo F. Obesidade em pacientes candidatos a cirurgia bariátrica. ACTA - Revista paulista de enfermagem, v. 22, p. $55-59,2009$. 
DINIZ, M. T. C., \& MACIANTE, B. A. Histórico: cirurgia bariátrica e metabólica. In: M. T. C. Diniz. (Org.). Cirurgia bariátrica e metabólica: abordagem multidisciplinar. São Paulo: Atheneu. 2012.

FABRICATORE, A.N., WADDEN, T.A., Psychological functioning of obese individuals. Diabetes Spectrum, v. 16, p. $246-251,2003$.

FANDIÑO, J., BENCHIMOL, A.K., COUTINHO, W.F., APPOLINÁRIO, J.C. Cirurgia bariátrica: aspectos clínicos-cirúrgicos e psiquiátricos. Revista Psiquiátrica do Rio Grande do Sul, v. 26, p. 47 - 51, jan./abr., 2004.

FISCHER, M., GOLDEN, N.H., KATZMAN, D.K., KREIPE, R.E., REES, J., SHEBENDACH, J., SIGMAN, G., AMMERMAN, S., HOBEREMAN, H.M. Eating disorders in adolescents: a background paper. Journal of Adolescent Health Care, v.16, n.6, p.420-437, New York, 1995.

FLANDRIN, Jean-Louis; MONTANARI, Mássimo. História da Alimentação. São Paulo: Estação Liberdade, 1998.

FRANQUES. Sobre o Comportamento e cognição. In: Arruda RTC (ed.) Obesidade mórbida e intervenção, ESETEC Editores Associados, p. 335, 2003.

FLORES, Carolinas Aita. Avaliação psicológica para cirurgia bariátrica: prática atuais. Porto Alegre. 2014.

GARROW, J. S., WEBSTER, J. Quetelet's Index (W/H2) as a measure of fatness. International Journal of Obesity. 1985.

GARRIDO Júnior, A.B. Cirurgia da obesidade. Atheneu. São Paulo. 2003.

GAYOSO, M. H., FONSECA, A., SPINA, L. D. C. \& EKSTERMAN, L. F. Obesidade: Epidemiologia, Fisiopatologia e Avaliação Clínica. ARS CVRANDI. 1999. 
GHONEIM M.M.; O’HARA M.W. Depressão pós-operatórias e complicações: uma visão geral. BMC Surgery, v. 16, fev., Iowa. EUA. 2016.

HERPERTZ, Stephan, MÜLLER, Astrid, BURGMER, Ramona, CROSBY, Ross D., ZWAAN, Martina, TANJA, Legenbauer. Health-related quality of life and psychological functioning 9 years restrictive surgical treatment for obesity. Surgery for obesity and related diseases, v. 11, p. 1361 - 1370, nov./dez., 2015.

HSU, L.K. et al. Nonsurgical factors that influence the outcome of bariatric surgery: a review. Psychosomatic Medicine, v. 60, n. 3, p. 338 - 346,1998.

LEAL, Cristiano W. BALDIN, Nelma. O impacto emocional da cirurgia bariátrica em pacientes com obesidade mórbida. Revista Psiquiátrica. RS. 2007.

LEITE, L.D., ROCHA, E.D.M., NETO, J.B. Obesidade: uma doença inflamatória. Revista Ciência e Saúde, Porto Alegre, v. 2, n. 2, p. 85-95, jul./dez. 2009.

MAGDALENO JR., Ronis; CHAIM, Elinton Adami; TURATO, Egberto Ribeiro. Características psicológicas de pacientes submetidos a cirurgia bariátrica. Rev. Psiquiátrica do Rio Grande do Sul, Porto Alegre, v. 31, n. 1, p. 73-78, 2009. Disponível em: $<$ http://www.scielo.br/scielo.php?script=sci_arttext\&pid=S0101-81082009000100013\&lng=e n\&nrm=iso $>$.Acesso em 13 nov. 2019.

MARCELINO, L.F. e PATRÍCIO, Z.M. A complexidade da obesidade e o processo de viver após a cirurgia bariátrica: uma questão de saúde coletiva. Ciência e saúde coletiva. 2011.

MARIANO, Maria L.L., MONTEIRO, Claudia S., PAULA, Maria A. B., Cirurgia Bariátrica: repercussões na vida laboral do obeso. Revista Gaúcha de Enfermagem,Porto Alegre, v. 34, p. $38-45,2013$. 
MATINI, Diana, JOLFAEI, Atefeh G., PAZOUKI, Abdolreza, PISHGAHROUDSARI, Mohadeseh, ENTESHAM, Mehdi. Medical Journal of The Islamic Republic of Iran, v. 28, p. $01-07$, out., 2014.

MATTOS, Maria Isabel Perez. Os transtornos alimentares e a obesidade numa perspectiva contemporânea: psicanálise e interdisciplinaridade. Contemporânea - Psicanálise e Transdisciplinaridade. Porto Alegre. RS. 2007.

MELO, M.E. Os números da obesidade no Brasil: VIGITEL 2009 e POF 2008 - 2009. 2009.

MELO, M.E., MANCINI M.C. Obesidade como diagnosticar e tratar. Revista Brasileira de Medicina. São Paulo: Moreira Jr., 2009.

MELlO FILHO, J. Grupo e Corpo: psicoterapia de grupo com pacientes somáticos. 1. Ed. Porto Alegre: Artes Médicas., 2000.

MINISTÉRIO DA SAÚDE. Coordenação-Geral da Política de Alimentação e Nutrição (CGPAN). Prevalência de déficit de peso e excesso de peso na população com 20 ou mais anos de idade, por sexo. Brasil - períodos 1974-1975, 1989 e 2002-2003. Brasília: Ministério da $2007 . \quad$ Saúde, Disponível $<$ http://dtr2004.saude.gov.br/nutricao/boletim_sisvan/documentos/>. Acesso em: 10. out. 2019.

OLGUIN, Pablo; CARVAJAL, Daniel; FUENTES, Manuel S. Patologia psiquiátrica y cirurgia bariátrica. Revista Chilena de Cirurgia. Chile. V. 67, n. 4, p. 441 - 447, ago., 2014.

OLIVEIRA, J.H.A. Aspectos psicológicos de obesos graus III antes e depois da cirurgia bariátrica. 2006. Tese de doutorado, Campinas, São Paulo. 2006.

ORGANIZAÇÃO MUNDIAL DE SAÚDE (OMS). Doenças não-transmissíveis: estratégia regional para $2000 \quad$ 2010. Disponível 
$<$ www.afro.who.int/textonly/press/portuguese/2000/rc/rc5006.html > Acesso em: 13. set. 2019.

PEREIRA, J.A. Avaliação das modificações metabólicas durante e após o emagrecimento em pacientes portadores de obesidade classe III, submetidos à cirurgia de gastroplastia vertical com bandagem e derivação gastro-jejunal. Tese (Doutorado), São Paulo. 2003.

PEREIRA, Érico Felden; TEIXEIRA, Clarissa Stefani; SANTOS, Anderlei dos. Qualidade de vida: abordagens, conceitos e avaliação. Revista brasasileira de educação física e esporte, São Paulo, v. 26, n. 2, p. 241-250, jun. 2012. Disponível em: $<$ http://www.scielo.br/scielo.php?script=sci_arttext\&pid=S180755092012000200007\&lng $=$ pt\&nrm=iso $>$. Acesso em: 13 nov. 2019.

PORIES, W.J.; JOSEPH, E.B.- Surgery for obesity: procedures and weight loss. In: Fairbuirn \& Brownell (eds) Eating disorders and obesity, 2. ed., New York, p. 562-7, 2003.

PRUZINSKY, T., EDGERDON, M. Body image change is cosmetic plastic surgery. In T. F. Cash \& T. Pruzinsky (Orgs.), Body images development, deviance, and change. P. 217 236. Nova Iorque: The Guilford Press. 1990.

PUCCI, G.C., RECH, C.R., FERMINO, R.C., REIS, R.S. Associação entre atividade física e qualidade de vida em adultos. Rev. Saúde Pública. v. 46, p.166 - 179, 2012.

REPETTO, G., RIZZOLLI, J., BONATTO, C. Prevalência, riscos e soluções na obesidade e sobrepeso: here, there and everywhere. Arquivos Brasileiros de Endocrinologia e Metabologia, v. 47, p. $633-635,2003$.

REZENDE, F. R. Percepção da imagem corporal, resiliência e estratégias de coping em pacientes submetidos à cirurgia bariátrica. Dissertação (Mestrado). Faculdade de Filosofia, Ciências e Letras de Ribeirão Preto, Universidade de São Paulo, SP. 2011.

ROSEMBAUM, P. Definição do problema: obesidade. 2012. 
RUTLEDGE, Thomas, BRADEN, Abby L., WOODS, Gina, HERBST, Karen, GROESZ, Lisa M., SAVU, Michelle. Five-year changes in psychiatric treatment status and weight-related comorbidities following bariatric surgery in veteran population. Obesity Surgery, v. 22, p. 1734 - 1741, nov., 2012.

SABISTON Júnior D.C., Tratado de cirurgia: as bases biológicas da prática cirúrgica moderna. Rio de Janeiro: Guanabara Koogan. 1999.

SANTINI, S. Cultura corporal e qualidade de vida. Kinesis, v. 27, p. 116 - 186, Santa Maria. 2002.

SEGAL, A., FANDIÑO, J. Indicações e contraindicações para realização das operações bariátricas. Revista Brasileira de Psiquiatria, v. 24, p. 68 - 72. 2002.

SICHIERI, Rosely et al. Recomendações de alimentação e nutrição saudável para a população brasileira. Arquivos Brasileiros de Endocrinologia \& Metabologia, São Paulo, v. 44, n. 3, p. 227-232, jun. 2000. Disponível em: $<$ http://www.scielo.br/scielo.php?script=sci_arttext\&pid=S000427302000000300007\&lng=en \&nrm=iso $>$. Acesso em: 13 nov. 2019.

SIERZANTOWICZ, Regina; LEWKO, Jolanta; HADY, Razak; KIRPSZA, Bozena; TROCHIMOWICZ, Lech; DADAN, Jacek. Effect of BMI on quality of life and depression levels after bariatric surgery. Advances in Clinical and Experimental Medicine. Polônia, v. 26, p. 491-496, mai.jun., 2017.

SILVA, V. L. M. Obesidade: O que nós, psicólogos, podemos fazer? Sobre comportamento e cognição, 1 ed., v. 6. Santo André: Editores Associados, cap. 33, p. 265 - 270. 2001.

SILVA, R. S. Reganho de peso após anos ou mais de pós-operatório de by-pass gástrico em Y-de-Roux. Dissertação (Mestrado). Pontifícia Universidade Católica do Rio Grande do Sul. Porto Alegre. RS. Brasil. 2011. 
SIMON, G.E., KORFF, M.V., SAUDERS, K., MIGLORETTI, D.L. Association between obesity and psychiatry desorders in the US adult population. Archives General Psychiatry, v. 63 , p. $824-830,2006$.

Sociedade Brasileira de Cirurgia Bariátrica e Metabólica. Técnicas de cirurgia bariátrica no Brasil. Disponível em: http://www.sbcb.org.br/. Acesso em: 13 ago. 2019.

SOCKALINGAM, Sanjeev, WNUK, Susan, KANTAROVICH, Karin, MEANEY, Christopher, OKRAINEC, Allan, HAWA, Raed, CASSIN, Stephanie. Employment outcomes one year after bariatric surgery: the role of patient and psychosocial factors. Obesity Surgery, v. 25 , p. $514-522$, mar., 2015.

SOUZA L. J. et al. Prevalência de obesidade e fatores de risco cardiovascular. Rio de Janeiro. 2003.

SUPLICY, H. Avaliando a obesidade a sua comorbidade. Paraná. 2005.

TAE, Barbara; PELAGGI, E.R.; MOREIRA, G.J.; WAISBERG, Jacques; MATOS, L.Leandro; D’Elia, G. O impacto da cirurgia bariátrica nos sintomas depressivos e ansiosos, comportamento bulímico e na qualidade de vida. Revista do Colégio Brasileiro de Cirurgiões. Brasil, v.41, p. 155-160, mai./jun., 2014.

TAVARES, M.C.C. Imagem corporal: conceito e desenvolvimento. São Paulo: Manole. 2003.

TEICHMANN, L., OLINTO, M.T.A., COSTA, J.S.D., ZIEGLER, D. Fatores de risco associados ao sobrepeso e à obesidade em mulheres de São Leopoldo, RS Revista Brasileira de Epidemiologia, São Leopoldo, v.9, p. 360 - 373. 2006.

WANDERLEY, Emanuela N., FERREIRA, Vanessa A. Obesidade: uma perspectiva plural. Ciência e Saúde Coletiva. p. 185 - 194. Minas Gerais. 2007. 
WORLD HEALTH ORGANIZATION. Obesity: preventing and managing the global epidemic; Report of a WHO Consultation. Geneve: World Health Organization; 2000.

WORLD HEALTH ORGANIZATION. Obesity: preventing and managing the global epidemic. Report of a WHO consultation on Obesity. Geneva: WHO.2006 